\title{
V006 APPLICATION OF NEURAL NETWORKS AND COKRIGING FOR THE MAPPING OF A RESERVOIR PROPERTY
}

\author{
KATUHEI YOSHIOKA and NOBUSUKE SHIMADA \\ Japan National Oil Corp., Technology Research Centre, 1-2-2 Hamada, Mihama-ku, Chiba 261, Japan
}

This presentation introduces the case study of reservoir characterization in the offshore oil field. This field is in a dome approximately $7 \mathrm{~km}$ wide, superimposed on anticlinal structure. The two Jurassic zones comprise the main oil reservoirs in the field. The formation of these reservoirs consists mainly of carbonates, accompanied by subordinate anhydrite. The upper zone ('A') is separated from the lower ('B') by an anhydrite layer 20 feet thick.

The objective here is to characterize a reservoir property of the zone ' $A$ '. Three-Dimensional seismic data were acquired in 1994. Log data of 42 wells exist for a currently processed area. The processed seismic data does not have enough quality for an inversion process. The reasons are that the extracted seismic wavelets near the well locations are not stable, and the thickness of the reservoir unit is less than the seismic resolution.

We extracted more than 20 attributes from the interpreted horizon or from the time window based on it, and correlated them to the reservoir property, which is porosity-thickness $(\Sigma \phi h)$ in this study, derived from well $\log$ analysis. The result was that each of those seismic attributes had only weak cross-correlation (correlation coefficient is less than 0.5 ). Then, the neural networks technique was tried in order to utilize a set of attributes and get a better crosscorrelation. Here, the input of neural networks is a set of seismic attributes. The desired output is $\Sigma \phi h$. A similar method was introduced by Johnston (1993) trying sand percent areal prediction. Using the derived relationship, we estimated the spatial distributions and their uncertainties by cokriging.

The validity of the above method which is a combination of neural networks and cokriging was investigated by some cross-validation tests, compared to the other approaches. One of the cross-validation tests, hiding 27 deviation wells here, is summarized in Figure 1. Figure 1a, Figure 1c, and Figure 1e show the estimated $\Sigma \phi h$ distributions by kriging, neural networks, and cokriging using neural networks output, respectively. The crossplots between the actual data and the values which were estimated at the well locations by the above 3 methods are shown in Figure $1 b, d$, and $f$, respectively. The larger gray circles represent the hidden well data in each of the graphs of Figure 1. 
By conducting cross-validation tests, it was found that relatively large dispersion between the actual data and the estimation can occur in the case of applying only neural networks. Consequently, it seems that cokriging using neural networks output cannot always estimate precisely, compared with kriging using only well data. Furthermore, it is possible that the risk of evaluation error at unknown data points becomes larger, because standard deviations are less estimated in cokriging than in kriging. That is, neural networks can easily generate a strong cross-correlation using multi-variables, but they make it difficult to evaluate uncertainties because their physical relationships are left ambiguous.

\section{REFERENCES}

Johnston, D. H., 1993, Seismic attribute calibration using neural networks, the 63rd Annual Meeting of the Society of Exploration Geophysicists, Expanded Abstracts, p. 250-253.
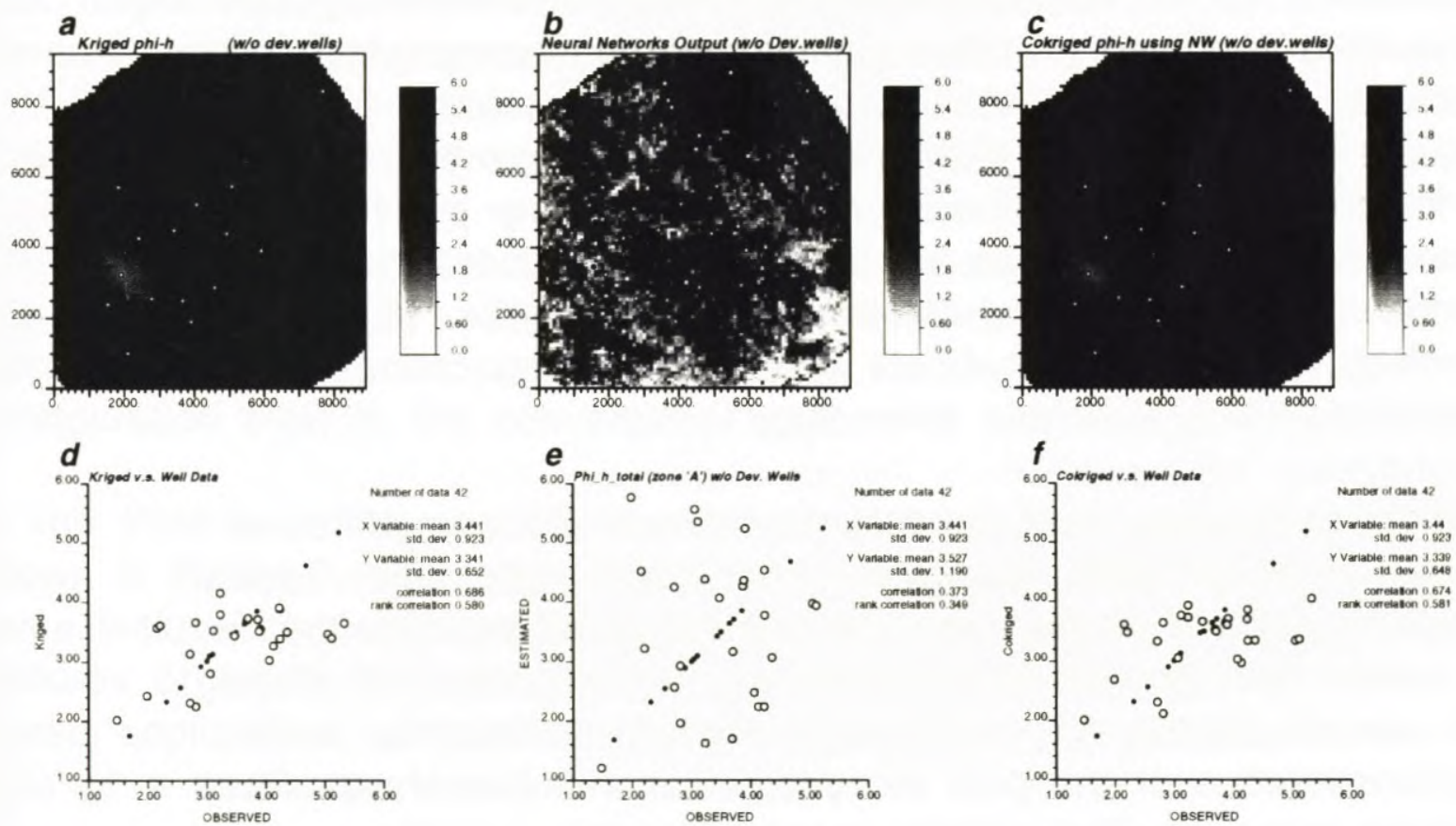

Figure 1. The cross-validation test hiding 27 deviation wells. (a) The $\Sigma \phi h$ distribution map by kriging and (d) its crossplot between the estimations and the actual data, (b) by neural networks and (e) its crossplot, and (c) by cokriging using neural networks output and (f) its crossplot. The gray circles indicate the hidden wells. The largest dispersion is seen in (b). The dispersion of (f) is almost same as that of (d). 